\title{
Magneto-optical Imaging Plate Using Bismuth-Substituted Iron Garnet Film Prepared by Metal-Organic Decomposition
}

\author{
Takayuki Ishibashi*, Gengjian Lou, Akira Meguro, \\ Takahiro Hashinaka, Michimasa Sasaki and Takao Nishi ${ }^{1}$ \\ Department of Materials Science and Technology, Nagaoka University of Technology, \\ 1603-1 Kamitomioka, Nagaoka, Niigata 940-2188, Japan \\ 'Department of Electronic Engineering, Kobe City College of Technology, \\ 8-3 Gakuen Higashimachi, Nishi-ku, Kobe, Hyogo 651-2102, Japan
}

(Received February 16, 2015; accepted May 21, 2015)

Key words: magneto-optical effect, magneto-optical imaging, bismuth-substituted iron garnet, metal-organic decomposition

Magneto-optical (MO) imaging plates consisting of bismuth-substituted rareearth iron garnet (Bi:RIG) films were studied. Bi:RIG films were prepared both on gadolinium gallium garnet (GGG) single-crystal and glass substrates by metal-organic decomposition. MO properties, Faraday rotation hysteresis, and Faraday spectra were measured. The MO imaging of the distribution of magnetic flux straying from magnetic videotapes was demonstrated using Bi:RIG films developed in this study.

\section{Introduction}

A magnetic imaging technique utilizing magneto-optical (MO) imaging plates has been attracting attention, because it is more advantageous in terms of features, such as measurement time, resolution, operating temperature range, and quantitatively evaluated magnetic flux and/or currents flow ${ }^{(1-6)}$ as compared with recently developed magnetic microscopy techniques, such as magnetic force and superconducting quantum interference device (SQUID) microscopies. A Bi-substituted rare-earth iron garnet film is used as an MO imaging plate that enables the visualization of magnetic fluxes, because it exhibits a large Faraday rotation in the transmission.

The MO imaging technique was developed in 1990 by Japanese and Russian research groups at almost the same time to observe stray fields induced by currents flowing in superconducting samples. ${ }^{(3,4)}$ The spatial resolution of this technique was further improved by using garnet films with in-plane magnetic anisotropy. The most impressive report proving the ability of MO imaging was that on a real-time observation of single vortices in a $\mathrm{NbSe}_{2}$ single crystal.(1,2)

*Corresponding author: e-mail: t_bashi@mst.nagaokaut.ac.jp 
The MO imaging technique, however, has not become popular, although it is a simple technique using a conventional polarization microscope as shown in Fig. 1. The main reason for this is that only few research groups can grow Bi-substituted iron garnet films with in-plane magnetic anisotropy; these films are typically grown by liquid phase epitaxy (LPE). ${ }^{(2,3)}$ To solve this problem, we have developed a metal-organic decomposition (MOD) method to prepare Bi-substituted rare-earth iron garnet (Bi:RIG) films. ${ }^{\left({ }^{8}\right)}$ Note that the MOD method has various advantages, such as the homogeneity of thin films, the controllability of the composition, and the formation of the films over a large area.

We have already reported that Bi:RIG films with high Bi substitution up to $x=3$ were successfully prepared on both gadolinium gallium garnet (GGG) single-crystal and glass substrates. $^{(5-10)}$ We suggest that these Bi:RIG films are suitable for use as MO indicator films in magnetic imaging. In this paper, we report on Bi:RIG films prepared by MOD and MO imaging using these Bi:RIG films.

\section{2. $B i_{x}: \mathbf{R}_{3-x}$ IG Films for MO Imaging Plate}

\subsection{Preparation of Bi:RIG films by $M O D$}

Bi:RIG films ( $\mathrm{R}=\mathrm{Y}, \mathrm{Nd}, \mathrm{Gd})$ were prepared by MOD. Figure 2 shows the MOD process consisting of spin coating of MOD solution (3000 rpm, $60 \mathrm{~s})$, drying $\left(100{ }^{\circ} \mathrm{C}, 10\right.$ $-30 \mathrm{~min}$ ), preannealing $\left(450{ }^{\circ} \mathrm{C}, 10-30 \mathrm{~min}\right)$, and annealing for crystallization $\left(700{ }^{\circ} \mathrm{C}\right.$, $3 \mathrm{~h}$ ). Chemical compositions of Bi:RIG films discussed in this paper are $\mathrm{Y}_{3-x} \mathrm{Bi}_{x} \mathrm{Fe}_{5} \mathrm{O}_{12}$ $(x=1-3)$ and $\mathrm{Nd}_{3-x} \mathrm{Bi}_{x} \mathrm{Fe}_{4} \mathrm{GaO}_{12}(x=1,2.5)$. Metal-organic solutions were prepared by Kojundo Chemical Co., Ltd. Bi:RIG films were directly grown on GGG substrates or $\mathrm{Nd}_{2} \mathrm{BiFe}_{4} \mathrm{GaO}_{12}$ buffer layers prepared on glass substrates. The thickness of the buffer layer on the glass (Corning Eagle XG) substrates was $120 \mathrm{~nm}$. The typical thickness of Bi:RIG films was $200 \mathrm{~nm}$.
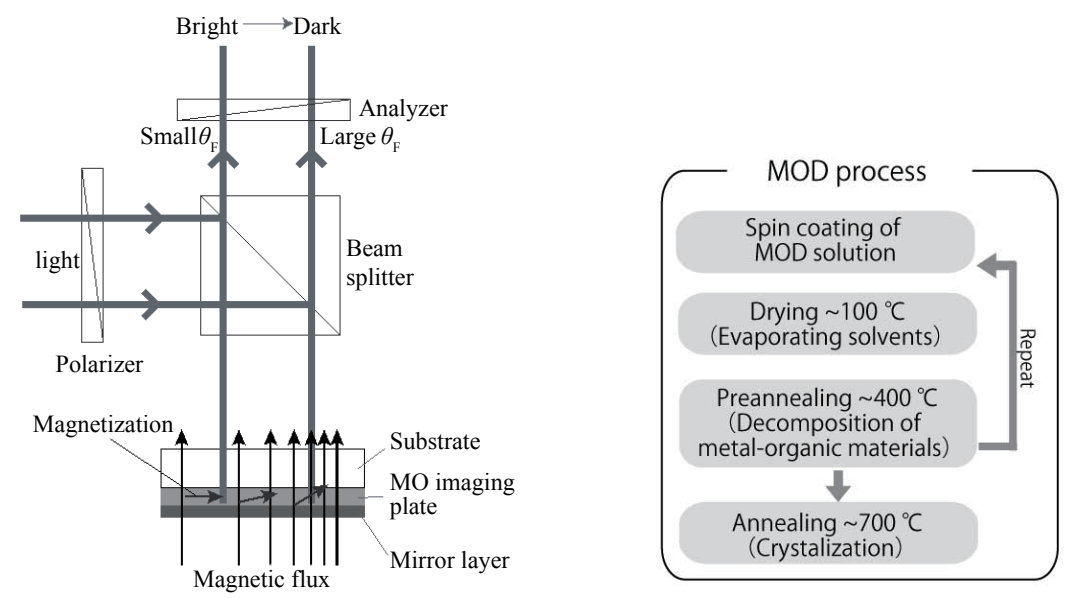

Fig. 1 (left). Schematic drawing of magnetic imaging using an MO imaging plate. The brightness in the image varies depending on the Faraday rotation angle $\theta_{\mathrm{F}}$ induced by a magnetic flux.

Fig. 2 (right). The MOD process consists of spin coating, drying, preannealing, and annealing; spin coating and preannealing are repeated several times to obtain the desired thickness. 


\subsection{Characterization of Bi:RIG films}

Figures 3(a) and 3(b) show X-ray diffraction (XRD) patterns of $\mathrm{Y}_{0.5} \mathrm{Bi}_{2.5} \mathrm{Fe}_{5} \mathrm{O}_{12}$ (YBIG) and $\mathrm{Bi}_{3} \mathrm{Fe}_{5} \mathrm{O}_{12}$ (BIG) films prepared on GGG (001). Only 004 and 008 peaks were observed to the left of those of the GGG substrates for both YBIG and BIG films, indicating that the YBIG and BIG films were grown on the substrate along the 001 direction. In other cases with different bismuth compositions of $x=0-3$, we also obtained a single phase of garnet films grown along the substrate orientation. On the other hand, polycrystalline garnet films were also successfully obtained on glass substrates. Figure 3(c) shows the XRD pattern of a BIG film prepared using a $\mathrm{Y}_{2} \mathrm{BiFe}_{5} \mathrm{O}_{12}$ buffer layer. We confirmed that most of the labeled peaks are due to polycrystalline BIG.

Faraday spectra of garnet films on GGG substrates are shown in Fig. 4, where $n$ denotes the number of repetitions of spin coating; each repetition corresponds to a thickness of $40 \mathrm{~nm}$. All spectra exhibited peaks between 500 and $530 \mathrm{~nm}$ owing to bismuth-substituted iron garnet, which corresponds to previous reports. ${ }^{(11,12)}$ We also found that the Faraday rotation angle increased with bismuth content, which also agrees well with previous reports. In our study, the maximum Faraday rotation reached $24 \mathrm{deg} /$ $\mu \mathrm{m}$ for the BIG film at a wavelength of $524 \mathrm{~nm}$, which is comparable to other reports. ${ }^{(13-15)}$

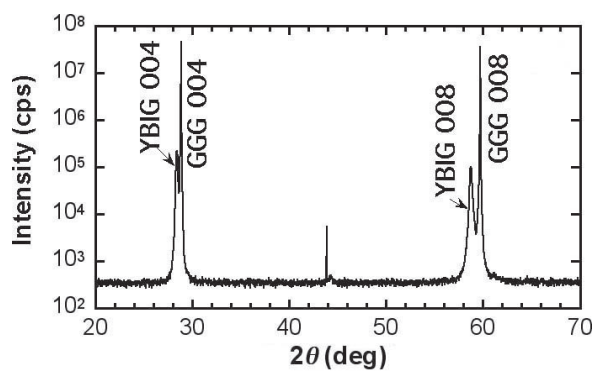

(a)

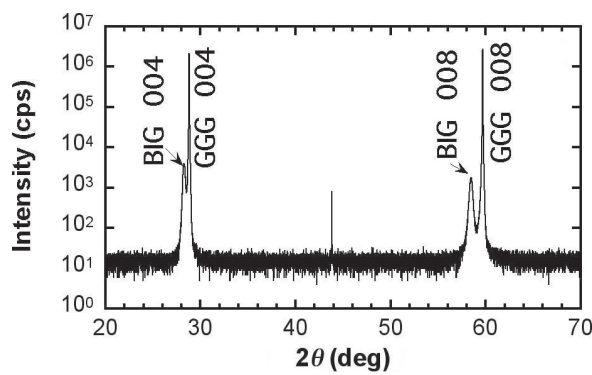

(b)

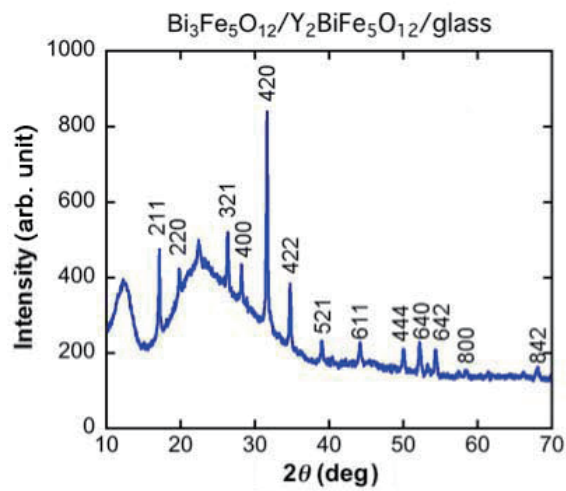

(c)

Fig. 3. (Color online) XRD patterns of (a) YBIG and (b) BIG films on GGG (001) substrates, and (c) BIG film on glass substrate. 


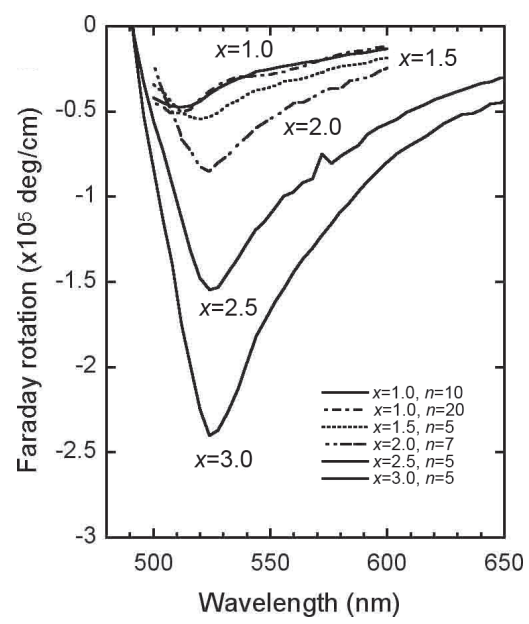

Fig. 4. Faraday spectra of $\mathrm{Bi}_{x}: Y_{3-x} \mathrm{IG}$ films prepared on GGG substrates with various bismuth compositions. ${ }^{(5)}$

\section{MO Imaging Using Bi:RIG Films}

Figure 5 shows the magnetic field dependence of the Faraday rotation angle for YBIG and BIG films with a thickness of $200 \mathrm{~nm}$ prepared on GGG substrates measured at a wavelength of $530 \mathrm{~nm}$ at room temperature and $3.8 \mathrm{~K}$. The Faraday rotation was measured in the reflection mode for a light reflected by an aluminum layer deposited on the YBIG and BIG films. The magnetic field dependences indicated that the YBIG film had the easy axis of magnetization in the in-plane direction, and had a small coercivity even at a low temperature of $3.8 \mathrm{~K}$. This linearity of the Faraday rotation of the YBIG film is considered to make the film suitable as an MO imaging plate that can be used for wide temperature ranges from room temperature to low temperatures. On the other hand, the BIG film did not show a rotation larger than that expected from the Faraday rotation spectra shown in Fig. 4 because of the large absorption at $530 \mathrm{~nm}$. The coercivity of the BIG film was also increased to approximately 200 Oe at $4 \mathrm{~K}$.

Figure 6 shows MO images of magnetic recording marks recorded in videotapes measured using the YBIG film, where wavelengths of the marks were 4 and $1.16 \mu \mathrm{m}$ for Figs. 6(a) and 6(b), respectively. Zigzag-shaped recording marks corresponding to magnetic signals are clearly observed, indicating that the spatial resolution in MO imaging using the YBIG film is higher than $0.6 \mu \mathrm{m}$, which is sufficient in comparison with that of an optical microscope.

\section{Large Bi:RIG Films}

For MO imaging plates with larger areas, glass substrates must be used, because the size of a GGG single crystal is limited to 1 for a (001) substrate. However, a Bi:RIG film with higher Bi content that exhibits a larger Faraday rotation cannot be grown directly on 


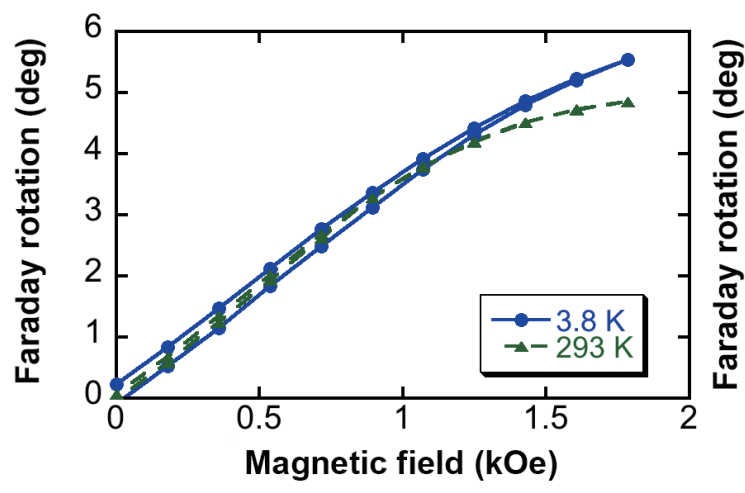

(a)

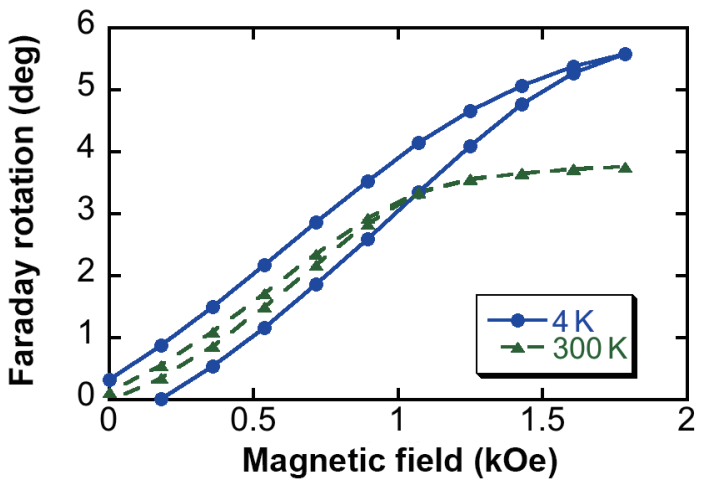

(b)

Fig. 5. (Color online) Magnetic field dependences of (a) BYIG and (b) BIG films on GGG (001) substrates measured at room temperature and low temperatures.
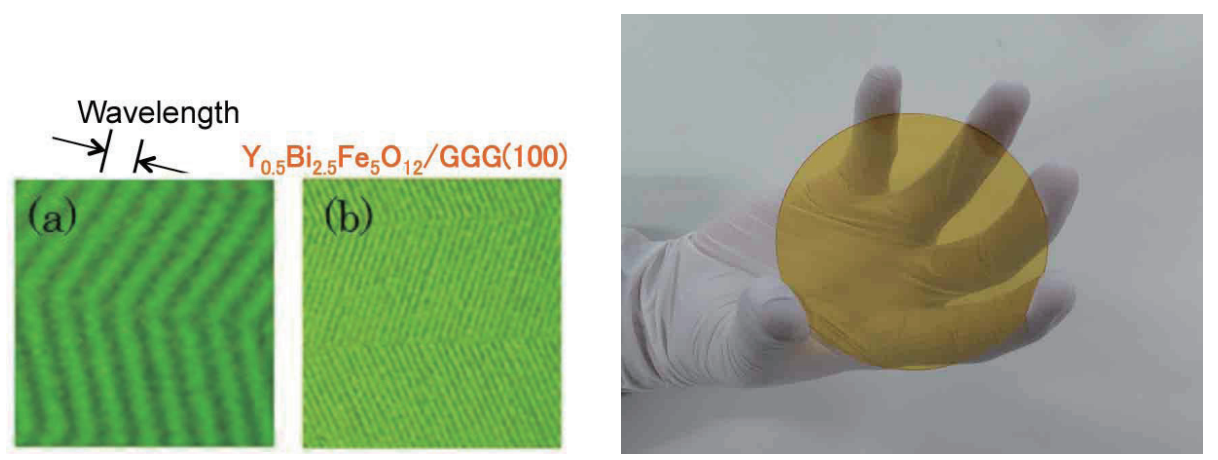

Fig. 6 (left). (Color online) MO images of magnetic recording marks recorded in videotapes with wavelengths of (a) 4 and (b) $1.16 \mu \mathrm{m} .^{(5)}$

Fig. 7 (right). (Color online) Photograph of $\mathrm{Nd}_{0.5} \mathrm{Bi}_{2.5} \mathrm{Fe}_{4} \mathrm{GaO}_{12}$ film prepared on a glass substrate of 3 inch diameter.

a glass substrate. We have succeeded in preparing Bi:RIG films with higher Bi content by using a buffer layer consisting of Bi:RIG films with low Bi content, $x=1$. $^{(10,12)}$ Figure 7 shows a photograph of a $\mathrm{Nd}_{0.5} \mathrm{Bi}_{2.5} \mathrm{Fe}_{4} \mathrm{GaO}_{12}$ film with a thickness of $200 \mathrm{~nm}$ prepared on a 3-inch glass substrate when a $\mathrm{Nd}_{2} \mathrm{BiFe}_{4} \mathrm{GaO}_{12}$ film with a thickness of $120 \mathrm{~nm}$ was used as a buffer layer. This film exhibited a large Faraday rotation of approximately 15 $\mathrm{deg} / \mu \mathrm{m}$ at $520 \mathrm{~nm}$. We confirmed that a smooth and homogeneous film was obtained.

We found that a $\mathrm{Nd}_{3-x} \mathrm{Bi}_{x} \mathrm{Fe}_{5-y} \mathrm{Ga}_{y} \mathrm{O}_{12}$ film is promising because its crystallinity and optical properties are higher than those of $\mathrm{Y}_{3-x} \mathrm{Bi}_{x} \mathrm{Fe}_{5-y} \mathrm{Ga}_{y} \mathrm{O}_{12} \cdot{ }^{(12)}$ We attribute these favorable properties to the similar ionic radii of $\mathrm{Nd}^{3+}$ and $\mathrm{Bi}^{3+}$, which makes $\mathrm{Bi}$ substitution easier than that in the case of $\mathrm{Y}_{3-x} \mathrm{Bi}_{x} \mathrm{Fe}_{5-y} \mathrm{Ga}_{y} \mathrm{O}_{12}$. 


\section{Conclusions}

Bi:RIG films were successfully prepared on both GGG single-crystal and glass substrates by MOD. Bi:RIG films with 001 orientation were obtained on the GGG single-crystal substrate, and polycrystalline Bi:RIG films were obtained on glass substrates. The films exhibited large Faraday rotation angles, which reached $25 \mathrm{deg} / \mu \mathrm{m}$ for BIG films, when $x=3$. YBIG films exhibited in-plane magnetic anisotropy both at room temperature and $3.8 \mathrm{~K}$, which makes them suitable for use as MO imaging plates. MO imaging was demonstrated for videotapes with magnetic recording marks with a bit length of $1.16 \mu \mathrm{m}$, indicating that the spatial resolution of the MO imaging plates was good enough for $\mathrm{MO}$ imaging. A large $\mathrm{MO}$ imaging plate consisting of a $\mathrm{Nd}_{0.5} \mathrm{Bi}_{2.5} \mathrm{Fe}_{4} \mathrm{GaO}_{12}$ film was fabricated on a 3-inch glass substrate.

\section{Acknowledgements}

This research was partly supported by the National Institute of Information and Communications Technology (NICT) and the Nanotechnology Platform at Yamaguchi University. We thank Prof. Nomura of the Shizuoka Institute of Science and Technology for measuring MO images of magnetic recording marks.

\section{References}

1 T. H. Johansen and D. V. Shantsev: Magneto-optical Imaging, Nato Science Series II Mathematics, Physics and Chemistry (Kluwer Academic Pub., 2004).

2 P. E. Goa, H. Hauglin, Å. A. F. Olsen, M. Baziljevich and T. H. Johansen: Rev. Sci. Instrum. 74 (2003) 141.

3 S. Gotoh, N. Koshizuka, M. Yoshida, M. Murakami and S. Tanaka: Jpn. J. Appl. Phys. 29 (1990) 1083.

4 M. V. Indenbom, N. N. Kolesnikov, M. P. Kulakov, I. G. Naumenko, V. I. Nikitenko, A. A. Polyanskii, N. F. Vershinin and V. K. Vlasko-Vlasov: Physica C 166 (1990) 486.

5 T. Kosaka, M. Naganuma, M. Aoyagi, T. Kobayasi, S. Niratisairak, T. Nomura and T. Ishibashi: J. Magn. Soc. Jpn. 35 (2011) 194.

6 N. Harada, Y. Goto, T. Yasuda, M. Tsuda and T. Hamajima: IEEE Trans. Appl. Supercond. 15 (2005) 3714.

7 T. Ishibashi, A. Mizusawa, M. Nagai, S. Shimizu, K.Sato, N. Togashi, T. Mogi, M. Houchido, H. Sano and K. Kuriyama: J. Appl. Phys. 97 (2005) 013516.

8 S. Ikehara, K. Wada, T. Kobayashi, S. Goto, T. Yoshida, T. Ishibashi and T. Nishi: J. Magn. Soc. Jpn. 36 (2012) 169.

9 T. Yoshida, K. Oishi, T. Nishi and T. Ishibashi: Eur. Phys. J. Web Conf. 75 (2014) 05009.

10 T. Ishibashi, T. Yoshida, T. Kobayashi, S. Ikehara and T. Nishi: J. Appl. Phys. 113 (2013) 212391JAP.

11 S. Wittekoek, T. J. A. Popma, J. M. Robertson and P. F. Bongers: Phys. Rev. B 12 (1975) 2777.

12 G. Lou, T. Yoshida and T. Ishibashi: J. Appl. Phys. 117 (2015) 17A749.

13 S. Kahl and A. M. Grishin: J. Magn. Magn. Mater. 278 (2004) 244.

14 N. Adachi, V. P. Denysenkov, S. I. Khartsev and A. M. Grishin: J. Appl. Phys. 88 (2000) 2734.

15 T. Okuda, T. Katayama, K. Satoh and H. Yamamoto: J. Appl. Phys. 69 (1991) 4580. 